\title{
A Robust BaCkground Removal ALgortiHMS USING FUZZY C-MEANS CLUSTERING
}

\author{
S.Lakshmi ${ }^{1}$ and Dr.V.Sankaranarayanan ${ }^{2}$ \\ ${ }^{1}$ Jeppiaar Engineering College, Chennai \\ lakshmi1503@gmail.com \\ ${ }^{2}$ Director, Crescent Engineering College, Chennai \\ sankarammu@yahoo.com
}

\begin{abstract}
Background subtraction is typically one of the first steps carried out in motion detection using static video cameras. This paper presents a novel method for background removal that processes only some pixels of each image. Some regions of interest of the objects in the image or frame are located with the help of edge detector. Once the region is detected only that area will be segmented instead of processing the whole image. This method achieves a significant reduction in computation time that can be used for subsequent image analysis. In this paper we detect the foreground object with the help of edge detector and combine the Fuzzy c-means clustering algorithm to segment the object by means of subtracting the current frame from the previous frame, the accurate background is identified.
\end{abstract}

\section{KEYWORDS}

Background removal, Surveillance, Image segmentation, Foreground detection, Background Subtraction, Fuzzy-C means Clustering

\section{INTRODUCTION}

Background subtraction is a process of extracting foreground objects in a particular image. The foreground object boundaries extraction reduces the amount of data to be processed and also provide important information about the object. If a car is going on the road, the car forms the foreground object and the road is considered as background.

Recognizing the moving objects from a video sequence is a critical task in many applications. A common approach is to perform background subtraction, which identifies moving objects from the portion of video frame. There are many challenging issues in designing a good background subtraction algorithm such as robust against changes in illumination and the shadows cast by moving objects.

\section{RELATED WORK}

Background subtraction is a standard method for the object localization in the video sequences especially for the surveilling applications where cameras are fixed [1].The straight forward technique of background subtraction is to just subtract previous frame with the current frame and threshold the result on each pixel. Several background subtraction algorithms have been proposed in the recent literature. All of these methods try to effectively estimate the background model from the temporal sequence of the frames. One of the simplest algorithms is frame differencing

DOI : 10.5121/ijnsa.2013.5207 
[2]. The current frame is subtracted from the previous frame. This method was extended such that the reference frame is obtained by averaging a period of frames also known as median filtering. [3][4][5]. But such a simple method fails when the object moves very slowly. It involves comparing a detected image with an estimate of the image if it contained no objects of interest.

The issues in background maintenances are introduced in[6]. Horprasert et al [7] proposed statistical color model to remove shadow with RGB. But in his method non-stationary background cannot be used.

The areas of the image plane where there is a significant difference between the observed and estimated images indicate the location of the objects of interest. The name background subtraction comes from the simple technique of subtracting the detected image from the estimated image and thresholding the result to generate the objects of interest. Dongxiang Zhou, et al. has presented their work on a novel background subtraction algorithm that is capable of detecting objects of interest while all pixels are in motion. This algorithm makes use of texture feature information instead of intensity information [8].The general requirements for a background removal algorithm are the accuracy in object contour detection (spatial accuracy) and temporal stability of the detection(temporal coherency)[9]. Gaussian Mixture Model is used to estimate the complicated background such as outdoor background with trees [10].

The paper is organized as follows. Section 3 lists the major issues in background subtraction. Section 4 presents the outline of the paper. The color image is converted into gray scale image in Section 5.Section 6 presents the idea of detecting the edges of the foreground object by using the algorithm suggested by the authors in [11]. Section 7 explains how the fuzzy c means clustering algorithm used for segmentation of the image using the detected edges of the object and also explains how to improve the accuracy of the segmentation for background subtraction. Section 8 shows experimental results in terms of accuracy and efficiency, when compared with former MOG [10] and CB [12] techniques. Section 9 concludes the paper.

\section{MAJOR ISSUES IN BACKGROUND SUBTRACTION}

The background subtraction is to differentiate moving foreground objects from the static or dynamic background. The important issues related to background subtraction is listed below. They are

- Camera motion due to environmental factors

- Different parts of the day

- Different whether condition

- As the sun sets, changes in the background over time

- Detection of building and different natural structures

- The objects may be occluded by other objects.

- Sometimes, background is not static due to uttering leaves and

- Due to noise in the image during image acquisition process

\section{SKETCH OF THE PAPER}

First, we have to take any video sequence for few minutes and then convert it into frames. Convert color image into grey scale image without losing the fine details of the given image. Then the boundaries of the foreground objects are extracted by using our edge detection 
algorithm. Thirdly, Fuzzy C-means clustering algorithm can be used to perform the accurate segmentation of the foreground object. Finally, the current frame pixel value will be subtracted from the previous frame to get the subtracted background. The outline of the paper is shown in Fig-4.1.

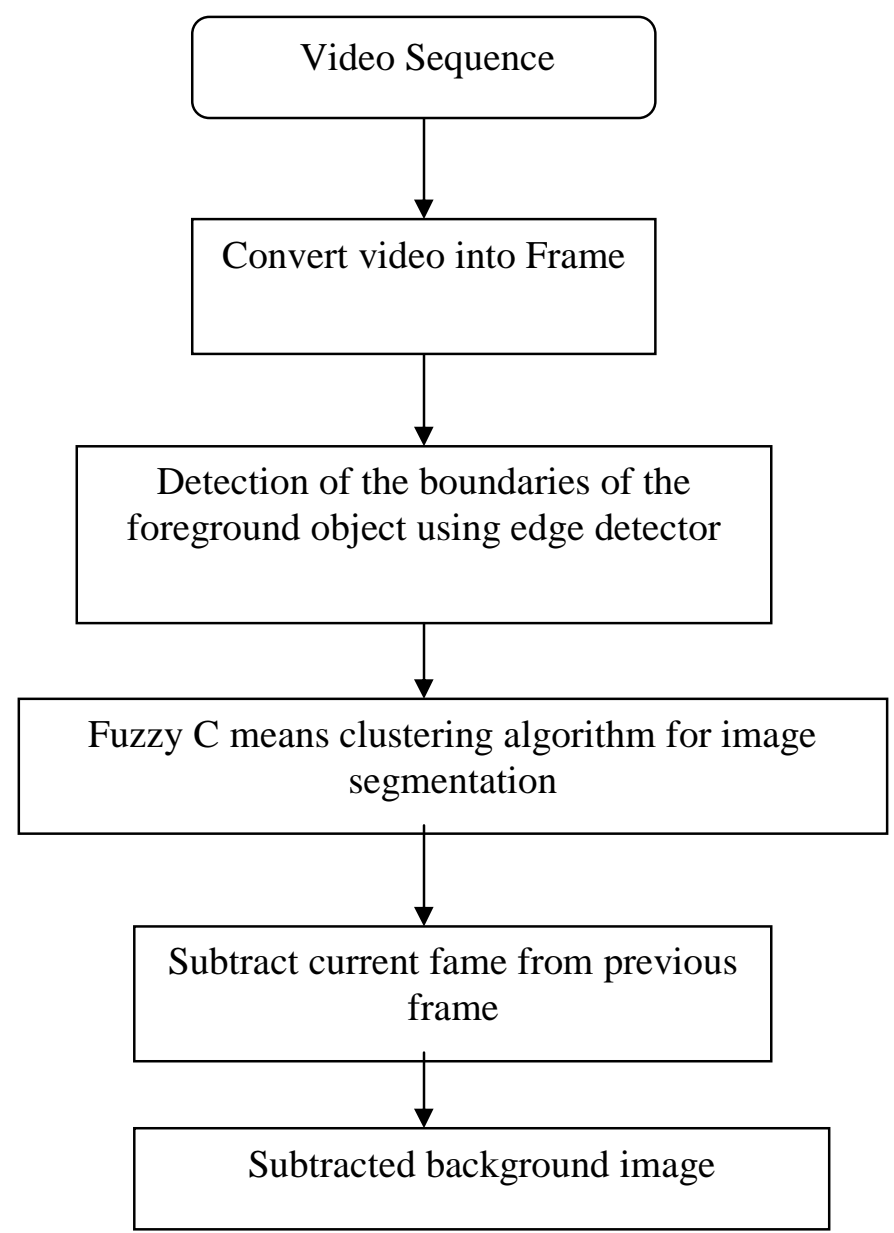

Fig-4.1-Outline of the paper

\section{CONVERSION OF COLOR IMAGE INTO GRAY SCALE IMAGE}

Generally images are represented in RGB color space. In the proposed algorithm, the color images need to be changed to gray ones. There are three eminent algorithms used to convert the color image into gray scale. They are lightness method, average method and luminosity method. Convert the color image into gray scale image by using any one of the methods. In this paper we have used average method for converting the color image into gray scale. In this average method the intensity of the each and every pixel is calculated using the formula

$$
\mathrm{I}=\frac{R+G+B}{3}
$$


For doing this conversion we have a function named rgb2gray (image) in matlab.We can also directly use this matlab function to convert the color image into gray scale image.

\section{EDGE DETECTION}

Digital image processing is the use of computer algorithms to perform image processing on digital images. Since the boundaries of the objects can have more information of the object than the object itself. For this reason edge detection plays a major role in computer vision. Hence, it is necessary to extract the fine details of the objects.

First, we have to apply the edge detection algorithm presented by the authors in [11], for detecting the edges of an image or frame. The aim of the edge detection is to eliminate the unwanted data to be processed and also to reduce the processing time by storing very important information.

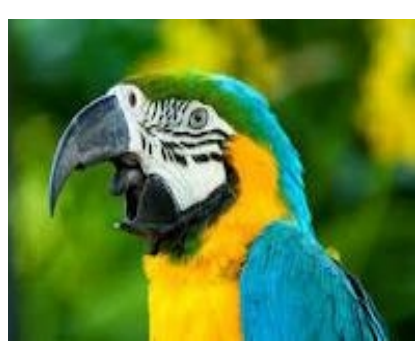

Fig.6.1.1

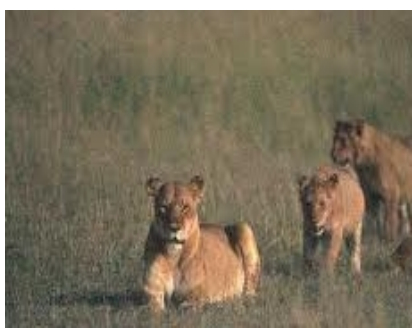

Fig.6.2.1

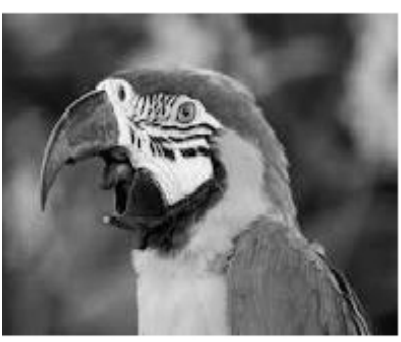

Fig.6.1.2

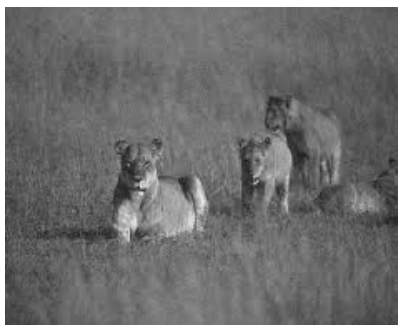

Fig.6.2.2

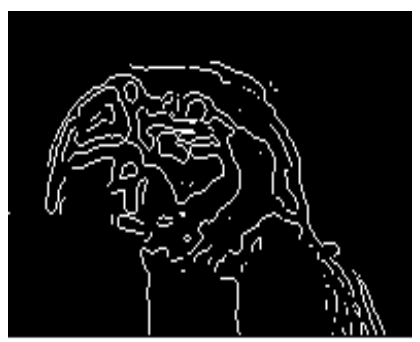

Fig.6.1.3

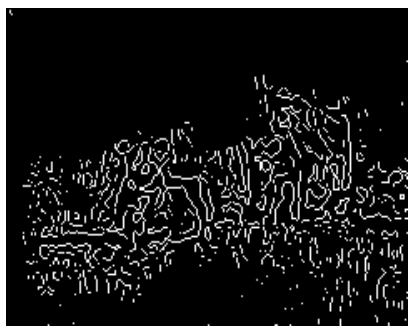

Fig.6.2.3

Fig.6.1.1 and 6.2.1 are original images;

Fig.6.1.2 and 6.2.2 are the gray scale images

Fig.6.1.3 and 6.2.3 are the detected edges of the given original image

\section{FUZZY C MEANS CLUSTERING}

The need of adapting efficient clustering algorithm increases in critical applications. Fuzzy CMean clustering algorithm is a way to show how data can be classified and clustered in organization or in any application [13]. It was developed by Dunn [14].In this paper, using Fuzzy c-means clustering algorithm background and foreground objects are segmented from the image or frames. This algorithm mainly helps to segment the pixels whether it belongs to background or foreground. The number of clusters is created based on the number of objects in the frames. Applying this fuzzy c means clustering algorithm centroid will be selected. First the centroid is 
chosen randomly based on the mean of the pixels. The correct centroid will be calculated after finding the degree of pixel using several iterations.

In this paper fuzzy c-means clustering method is used for choosing the centroid based on the pixels and the detected edges using the novel edge detection algorithm [11].The following algorithm shows how the fuzzy c-mean clustering technique can be used to segment the foreground object from the given image/frame.

\section{Algorithm}

Input: the boundaries of the object after applying the edge detection algorithm

Output: the segmented image by applying fuzzy c-means clustering algorithm

Step 1: Consider all the pixels at the left vertical axis of the frame.

Step 2: Scan every pixel in horizontal direction until it intersects with the edge pixel which has been detected during edge detection process.

Step 3: Store the value of the edge pixel and continue the process until other edge pixel is found and name those pixels as a, b and so on which gives the boundaries of the object.

Step 4: Continue the scanning process until reach the last pixel in the right vertical axis of the frame.

Step 5: If there is no edge pixel is found then those pixels are considered as background pixels.

Step 6: Calculate the mid point between a pixel in edge a and a pixel in edge $b$ using the formula

$$
\text { Midpoint }_{(\mathrm{a}, \mathrm{b})}=\frac{\left(x_{a}, y_{a}\right)+\left(x_{b}, y_{b}\right)}{2}
$$

where $\left(\mathrm{x}_{\mathrm{a}}, \mathrm{y}_{\mathrm{a}}\right)$ is the coordinate of a pixel in edge a and $\left(\mathrm{x}_{\mathrm{b}}, \mathrm{y}_{\mathrm{b}}\right)$ is the coordinate of a pixel in edge $\mathrm{b}$.

Step 7: Identify the mid points for all edge pixels by applying the Midpoint formula.

Step 8: Connect all the mid points column wise.

Step 9: Locate the column which contains more number of midpoints and mark the first and last midpoint in that column.

Step10: Calculate the midpoint for that column using the midpoint formula which acts as the centroid for the object in the frame or image.

The foreground objects in the frame or image are segmented by applying this algorithm. The detected edge pixels during edge detection process in the first stage are really very useful to segment the objects exactly. 
The background is subtracted by applying the above method to every frame of the video and the difference will be calculated between the previous and current frame as follows.

\section{Pixel coordinates $(\mathrm{x}, \mathrm{y})=$ Previous frame - Current frame}

The subtracted background for the given input images Fig-6.1.1 and Fig-6.2.1 are shown in the figures Fig-7.1 and Fig-7.2.

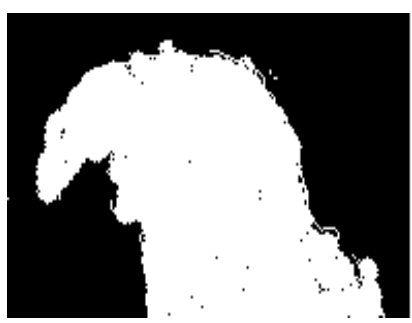

Fig.7.1

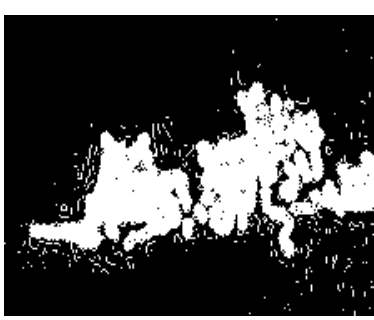

Fig.7.2

\section{RESULTS AND DISCUSSIONS}

We applied the proposed algorithm to various video streams. Fig-6.1.1 and Fig-6.2.1 shows the original image and Fig-6.1.2 and Fig-6.2.2 shows the grayscale images of the given original image. The detected edges of the original image after applying the edge detection algorithm are shown in Fig-6.1.3 and Fig-6.2.3. Apparently, the detected edges of the image or frame can remove the most of the noise and significantly enhance the detection precision.

Fig.7.1 and Fig.7.2 are the segmented images of the given input image after applying the fuzzy CMeans clustering algorithm. Several metrics are available to test the accuracy and performance of our method. We used the metric in terms of FP rate, TP rate, Precision and similarity.

$$
\begin{aligned}
& \text { FP rate }=\frac{f p}{f p+t n} \\
& \text { TP rate }=\frac{t p}{t p+t n} \\
& \text { Precision }=\frac{t p}{t p+f p} \\
& \text { Similarity }=\frac{t p}{t p+f p+f n} \\
& \text { Recall }=\frac{t p}{t p+f n}
\end{aligned}
$$

where tp denotes the total number of true positives;

tn denotes the number of true negative $\mathrm{fp}$ denotes the number of false positives fn denotes the number of false negatives 
$(\mathrm{tp}+\mathrm{fn})$ indicates the total number of pixels presented in the foreground and

$(\mathrm{fp}+\mathrm{tn})$ indicates the total number of pixels presented in the background.

We have tested the results for every 40 frames.

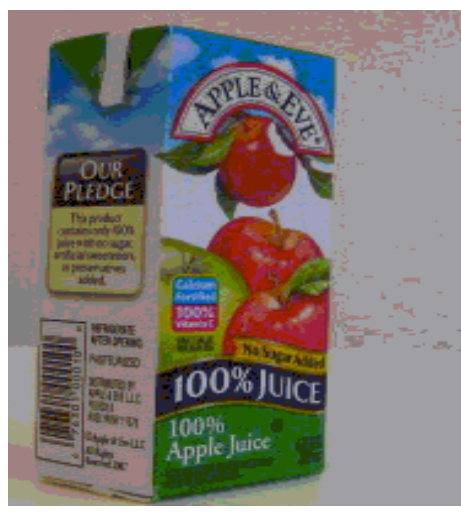

Fig.8.1

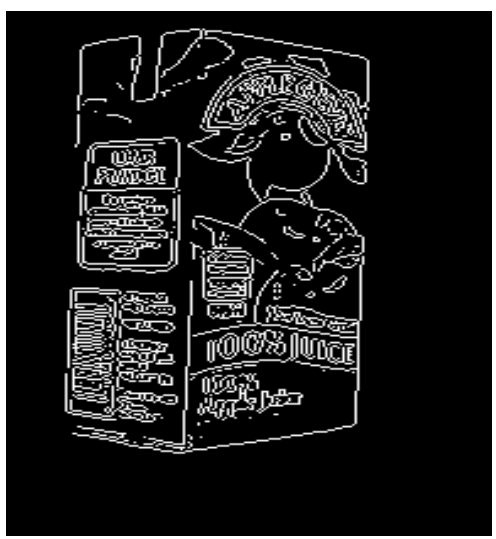

Fig.8.3

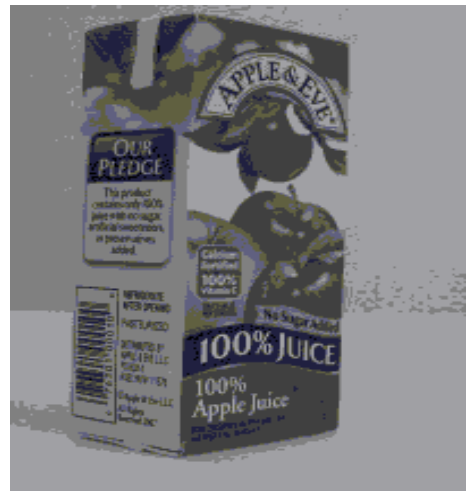

Fig.8.2

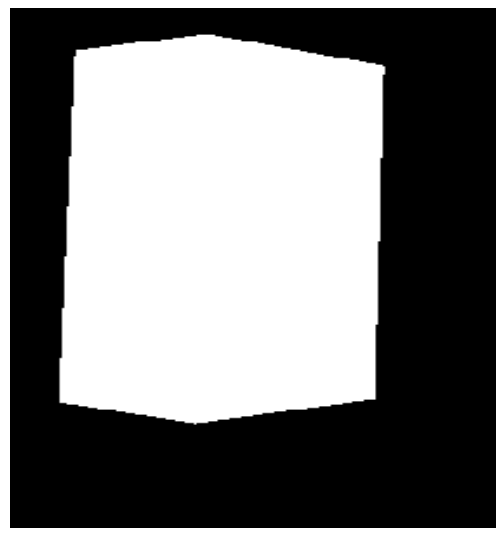

Fig.8.4

The proposed work is evaluated by using another frame Fig.8.1.The gray scale image of the Fig.8.1 is given in Fig.8.2. The Fig.8.3 shows the detected edges of the given original image and the Fig.8.4 shows the final result of background subtraction. Fig 8.5 is the original image of another example and Fig.8.6 is the resultant image of our background subtraction algorithm. 
International Journal of Network Security \& Its Applications (IJNSA), Vol.5, No.2, March 2013

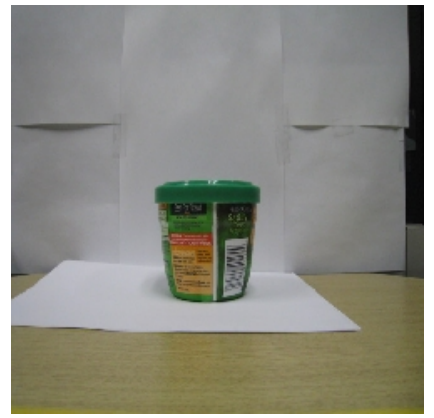

Fig.8.5

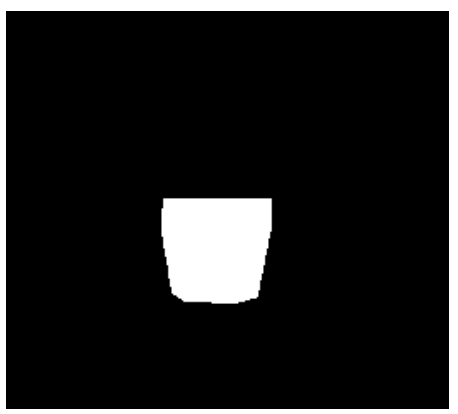

Fig.8.6

The accuracy of the proposed method has been evaluated and the results are compared and tabulated with the previous methods in Table 1. The results are shown diagrammatically in Fig8.7 .

TABLE 1.PERFORMANCE EVALUATION

\begin{tabular}{|c|c|c|c|c|}
\hline & FP & TP & Precision & Similarity \\
\hline MOG & 0.035 & 0.931 & 0.835 & 0.785 \\
\hline CB & 0.014 & 0.908 & 0.903 & 0.827 \\
\hline $\begin{array}{c}\text { Proposed } \\
\text { Method }\end{array}$ & 0.071 & 0.964 & 0.704 & 0.687 \\
\hline
\end{tabular}

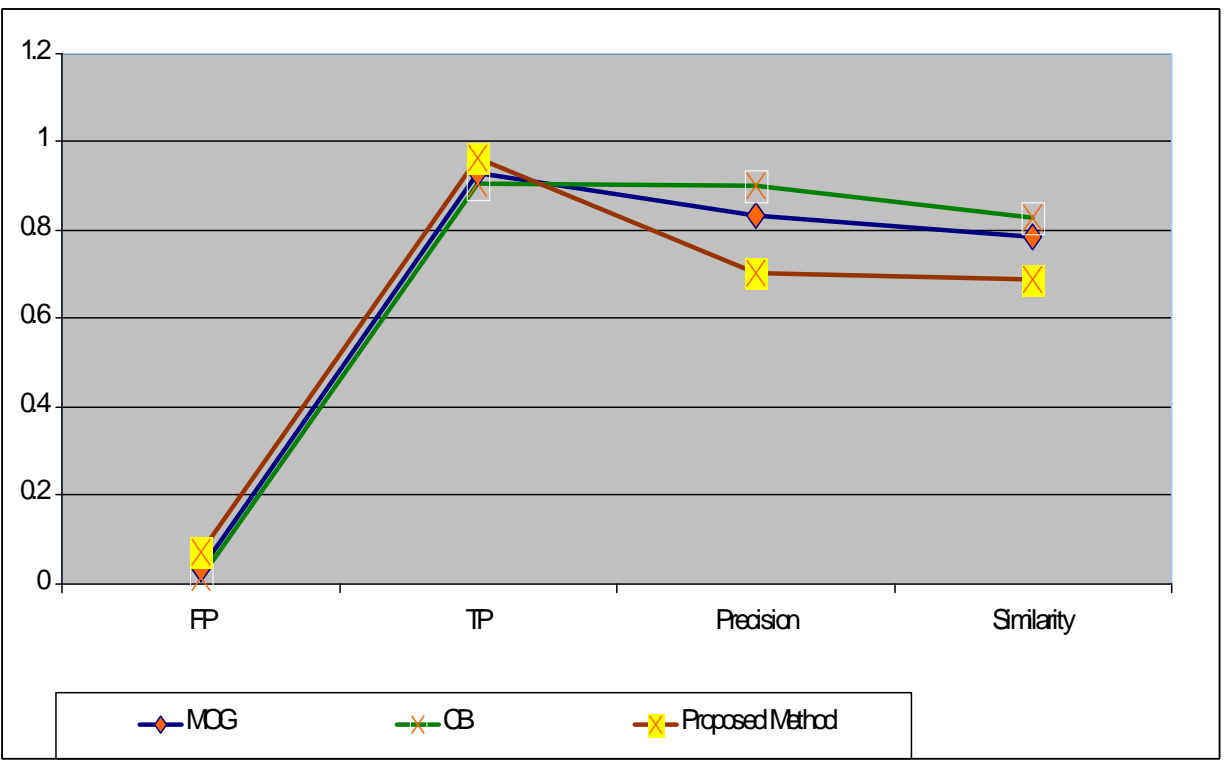

Fig. 8.7 Comparison of the proposed method with other methods 
International Journal of Network Security \& Its Applications (IJNSA), Vol.5, No.2, March 2013

Despite the true positives are more or less same with all the techniques, the precision and the similarity ratios are less in the proposed work. Hence, the proposed technique works better than other techniques.

\section{CONCLUSION}

A background subtraction method has been proposed by using edge detector and fuzzy c-means clustering algorithm. As documented in the experimental results, the proposed method provides high efficient for background subtraction which can be applied for vision based applications such as human motion analysis or surveillance system. Through this method, the execution speed is improved.

\section{REFERENCES}

[1] Gautam S. Thakur, Mohsen Ali, Pan Hui and Ahmed Helmy, (2012) "Comparing Background Subtraction Algorithms and Method of Car Counting", The Department of Computer and Information Science and Engineering, University of Florida, Gainesville.

[2] N.Friedman and S.Russel, [1997] "Image segmentation in video sequences: A probabilistic approach", in Proceedings of IEEE International Conference on Uncertainty in Artificial Intelligence, San Francisco, pp.175181.

[3] D.Hong and W.Woo,(2003) "A background subtraction for a vision-based user interface", in Proceedings of IEEE International Conference on Information, Communications and Signal Processing, Vol.1, pp.263-267.

[4] M.Harville,G.Gordon and J.Woodfill, "Foreground Segmentation using adaptive mixture models in color and depth", in Proceedings of IEEE Workshop on Detection and Recognition of Events in Video, Los Angeles, USA, pp.3-11, 2001.

[5] R.Cucchira,C.Grana, M.Piccardi, and A.Prati, "Detecting moving objects, ghosts and shadows in video streams," "IEEE Transactions on Pattern Analysis and Machine Intelligence,vol.25,no.10,pp.1337-1342,2003

[6] K. Toyama, J. Krumm, B. Brumitt, and B. Meyers, "Wallflower: principles and practice of background maintenance,” In Proc. IEEE Conf. Computer Vision, vol. 1, pp. 255-261, Sept. 1999

[7] T. Horprasert, D. Harwood, and L. S. Davis, "A statistical approach for real-time robust background subtraction and shadow detection,” IEEE ICCV Frame-Rate Applications Workshop, Kerkyra, Greece, Sept. 1999

[8] Dongxlang Zhou, Hong Zhang and Nilanjan Ray, "Texture Based Background Subtraction", Proc. IEEE Int. Conf. on Information and Automation, Zhangjiajie, China,pp.601-605,2008

[9] Shireen Y.Elhabian, Khaled M, El-Sayed and Sumaya H. Ahmed, Moving Object Detection in Spatial Domain using Background Removal Techniques - State of Art”, Recent Patents on Computer Science, pp-32-54, Bentham Science Publishers Ltd.,2008.

[10] C.Stauffer and W.E.L.Grimson, "Adaptive Background Mixture Models for Real-Time Tracking” in Proceedings of IEEE Conference on Computer Vision and Pattern Recognition, Fort Collins, Colorado, USA ,vol.2,pp.2462521999.

[11] S. Lakshmi and Dr.V.Sankaranarayanan, "A Novel Approach for Edge Detection”, IJCSNS International Journal of Computer Science and Network Security, Vol.10, No.4, April 2010.

[12] K. Kim, T.H. Chalidabhongse, D. Harwood, and L. Davis, "Real time foreground-background segmentation using codebook model,” Real-Time Imaging, vol. 11, no. 3, pp. 172-185, June. 2005.

[13] Bassam M,EI-Zaghmouri,Marwan A.Abu-Xanona, "Fuzzy C-Mean Clustering Algorithm Modification and Adaptation for Applications", World of Computer Science and Information Technology Journal(WCSIT), Vol2,/no.1,42-45,2012

[14] J.C.Dunn,"A Fuzzy Relative of the ISODATA Process and Its Use in Detecting Compact Well Separated Clusters", Journal of Cybernetics, pp:32 - 57, 1973. 